\title{
1 Effect of tilt angle on soiling in perpendicular wind
}

Benjamin Figgis $^{\mathrm{a}}{ }^{*}$, Dirk Goossens ${ }^{\mathrm{b}, \mathrm{c}}$, Bing Guo ${ }^{\mathrm{d}}$, Klemens Ilse $^{\mathrm{e}, \mathrm{f}}$

$4 \quad$ a Qatar Environment \& Energy Research Institute, HBKU, Doha, Qatar

${ }^{\mathrm{b}}$ ESAT/ELECTA KU Leuven \& EnergyVille, Kasteelpark Arenberg 10, 3001 Leuven, Belgium

${ }^{c}$ Geography and Tourism Research Group, KU Leuven Department of Earth and Environmental

7 Sciences, Celestijnenlaan 200E, 3001 Leuven, Belgium

8 d Texas A\&M University at Qatar, Doha, Qatar

$9{ }^{\mathrm{e}}$ Fraunhofer Center for Silicon Photovoltaics CSP, D-06120 Halle (Saale), Germany

$10{ }^{\mathrm{f}}$ Fraunhofer Institute for Microstructure of Materials and Systems IMWS, 06120 Halle (Saale),

11 Germany

*Contact. bfiggis@hbku.edu.qa

\section{Abstract}

Use of horizontal single-axis trackers in PV power plants is becoming more common, especially with the introduction of bifacial modules. This raises the possibility of combating soiling by adjusting the tracker tilt angle to minimize dust deposition or maximize wind cleaning, at night or when cloudy. In this study we experimentally investigated soiling of coupons tilted about an axis perpendicular to wind, using both an environmental wind tunnel and desert field tests. CFD modeling was also performed to help interpret the experimental results. In the field it was found that maximum deposition (total particles impacting the coupon) occurred when coupons were tilted around $45^{\circ}$ toward the wind. However maximum accumulation (particles remaining on the coupon) occurred at $22^{\circ}$ tilt away from the wind. The difference was due to a high fraction of particles detaching from coupons when they are tilted toward the wind, which raises flow velocity and shear rate near the surface. The results suggest that to minimize soiling at night, 1-axis PV trackers could be stowed at maximum tilt toward the wind.

27 Keywords: dust; tilt; soiling; desert; deposition; wind tunnel; CFD

\section{Introduction}

This study aimed to assess the potential to reduce soiling of 1-axis tracker PV systems, by investigating dust deposition and accumulation on tilted glass plates. Soiling of PV modules can greatly reduce their efficiency in desert environments, where there is much dust and little rain (Costa et al., 2016). The mechanics of dust particle deposition and detachment are controlled by airflow over a module's surface, which in turn is influenced by the module's tilt angle. This is especially of interest because of growing 
use of single-axis trackers in large-scale PV projects, which raises the possibility of using the tracker angle as a tool to minimize soiling.

In this study we used an environmental wind tunnel and outdoor field tests to measure soiling of tilted glass coupons, and CFD simulations to help explain the experimental results. The intention was to combine the advantages of each method: wind tunnels provide repeatable and comparable experimental data; field testing captures real-world environmental conditions and phenomena; and CFD provides detailed information on airflow characteristics close to the coupons.

The study focused on tilt axis perpendicular to the wind, i.e. the coupons faced toward or away from the wind. Although in the real world wind can come from any direction, the case of perpendicular airflow is especially interesting as in this orientation the PV tracker angle is expected to most influence the soiling rate. It focused on conditions of light or moderate wind (up to $\sim 5 \mathrm{~m} \cdot \mathrm{s}^{-1}$ ). Absent wind, soiling is governed by gravity settling of dust particles, and a module's soiling is simply proportional to the cosine of its tilt angle (Figgis et al., 2017a).

A further goal of the study was to distinguish between dust deposition and its accumulation, for the better understanding of soiling physics. To clarify the terminology herein:

- Deposition refers to all dust particles impacting the surface. In the field and wind-tunnel experiments it was measured as the dust captured by double-sided tape attached to the coupon. In CFD, it was measured by "freezing" particles when they contacted the surface

- Accumulation refers to the particles remaining on the surface at the end of the exposure (what is commonly thought of as "soiling"). In experiments it was measured as the dust remaining on the coupon's bare glass. It was not simulated in CFD because it is difficult to realistically model adhesion and removal of natural dust particles in outdoor conditions

- Detachment refers to the difference between deposition and accumulation, i.e. particles that impacted the surface but departed again before the end of the exposure, either by rebound (immediate departure) or resuspension (departure after some time).

In this report tilt angles are given relative to horizontal, positive toward the wind. For example, $45^{\circ}$ means tilted toward (facing) the oncoming wind, $0^{\circ}$ is horizontal, and $-45^{\circ}$ is tilted away from the wind (facing downwind). See Figure 1 for further illustration.

The effect of wind on PV soiling is complex. Wind increases both deposition of dust particles and their detachment from the surface. The balance between them is sensitive to many factors - geometry of the PV system, airflow characteristics (speed, direction, turbulence), dust characteristics (size, shape, composition), humidity, and other factors (Figgis et al., 2017a)(Javed et al., 2017)(Figgis et al., 2017b). As a result different reports, previously summarized (Figgis et al., 2017a), have demonstrated both netcleaning and net-soiling effects of wind. 
To understand the physics of soiling it is useful to isolate and vary one parameter while keeping others constant. Here we were interested in the effect of tilt angle on soiling, which meant minimizing variation in wind speed and direction. While there have been many field studies of soiling of PV modules and coupons at different tilt angles, those holding wind direction constant are scarce. One such report used a wind vane with a coupon tilted about an axis stream-wise to the wind (Biryukov, 1998), but none were found using a tilt axis perpendicular to the wind as in the present study. On the other hand numerous wind-tunnel studies have been performed (Goossens et al., 1993)(Smits K., Goossens, 1995)(Goossens, 2019). Zimon (Zimon, 1969) reported two wind-tunnel studies especially pertinent, on deposition of magnetite particles at tilts from $0^{\circ}$ to $90^{\circ}$, and resuspension of glass spheres from a steel surface at tilts spanning $360^{\circ}$. The results are discussed in section 3.2 and compared to present results. CFD has been used to simulate deposition of dust particles in many applications, including PV systems (Lu et al., 2016)( $\mathrm{Lu}$ and $\mathrm{Zhang}, 2019)(\mathrm{Lu}$ and Zhao, 2018). However it is difficult to predict detachment of dust from outdoor surfaces via computer simulation because it is sensitive to adhesion forces and particle shapes, which vary greatly in natural conditions (Ilse et al., 2018). Of course it is dust accumulation - rather than deposition - that is of practical interest to PV users. Although some CFD studies of PV soiling assumed that zero particles will be detached after deposition, a previous field study (Figgis et al., 2018) demonstrated that (in the particular environment) nearly $100 \%$ of impacting particles detached from a horizontal glass coupon when wind speed reached just $4 \mathrm{~m} \cdot \mathrm{s}^{-1}$.

To summarize the current state of research, wind-tunnel tests have provided useful information on dust deposition and detachment versus tilt angle; field experiments which isolate the effect of surface tilt in wind have been absent; and CFD has proved useful for detailed information on airflow characteristics near to surfaces which can help interpret experimental results.

\section{Methods}

\subsection{Wind-Tunnel Tests}

Wind-tunnel experiments were carried out in the closed-return wind tunnel of the Geography and Tourism Research Group of KU Leuven, Belgium. An abbreviated description of the wind-tunnel equipment and procedures is presented herein; further details are provided in the Supplementary Information. The tunnel included a dust-cloud producer that, in this study, dispersed Belgian Brabantian dust, which is representative of common deserts including Qatar (Péwé, 1981). The mass median particle diameter of airborne dust in the wind tunnel was $37 \mu \mathrm{m}$. However dust that accumulates on surfaces in wind is generally finer than the concurrent airborne dust, due to preferential resuspension of large particles (Figgis et al., 2017a)(Goossens, 2019). At $5 \mathrm{~m} . \mathrm{s}^{-1}$ wind speed (the target in the field tests) the average median diameter on coupons in the wind tunnel was $20 \mu \mathrm{m}$, corresponding adequately with $15 \mu \mathrm{m}$ for dust collected from PV modules at the Qatar field site (Figgis et al., 2017a).

An example of the wind tunnel set-up is shown in Figure 1. In each test run, a glass coupon $20 \times 20 \mathrm{~cm}$ was located in the center (laterally and vertically) of the tunnel. A strip of double-sided adhesive tape, 4 
$\mathrm{cm}$ wide, was attached along the stream-wise centerline of the coupon. Deposition was characterized by the amount of dust collected on the tape, while accumulation was characterized by the amount of dust collected on bare glass adjacent to the tape. A pair of BSNE dust collectors (Fryrear, 1986) flanking the coupon measured dust concentration during each test run, which was used to adjust the deposition and accumulation results for slight variations in dust concentration between test runs. Relative humidity was around $60 \%$ during the tests, and remained within the range $50-70 \%$.

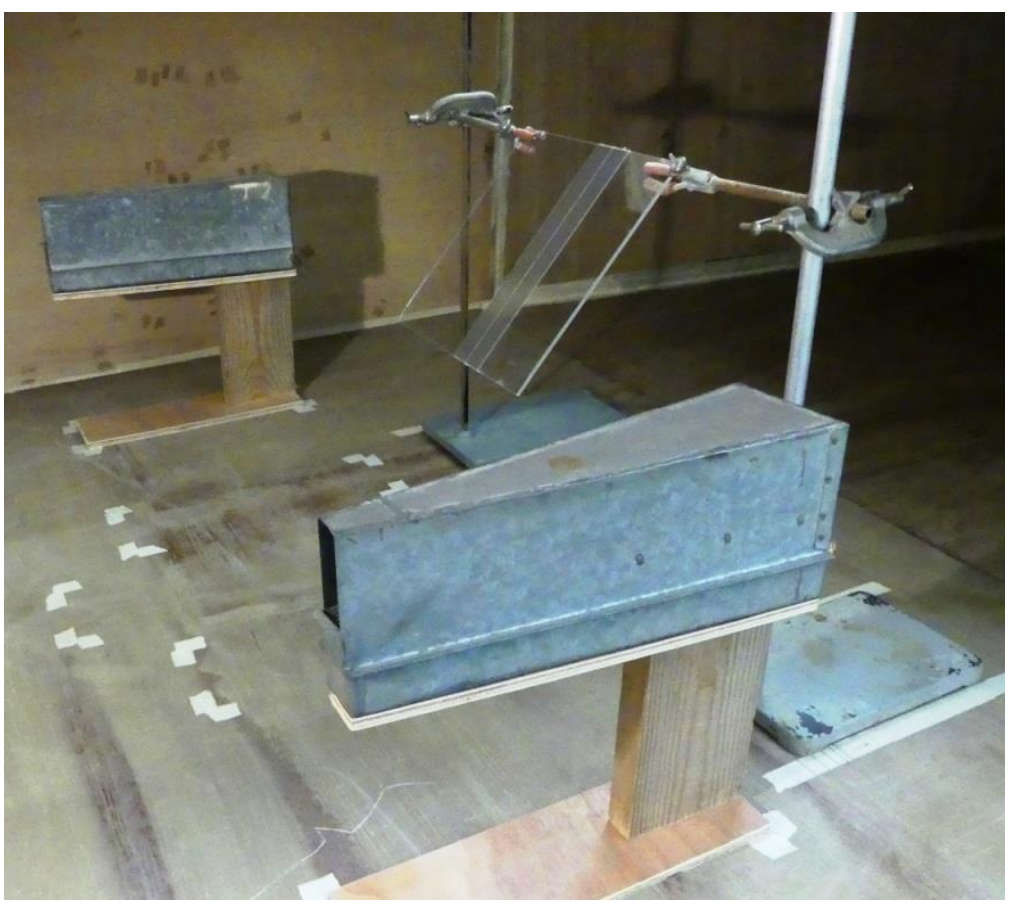

Figure 1. Set-up of wind tunnel tests. A glass coupon was placed the middle of the tunnel, with double-sided adhesive tape along its centerline. Wind direction was from the left, i.e. in this example the coupon is tilted "forward" (toward the wind), and its windward edge is to the lower left. The coupon is flanked by a pair of dust collectors used to normalize the soiling amounts between test runs.

Free-stream wind speeds for the wind-tunnel tests were set at 1, 2, 3, $4 \mathrm{~m} \cdot \mathrm{s}^{-1}$. The coupon was outside the boundary layer of the wind-tunnel walls at all speeds. The maximum coupon tilt in the wind tunnel was $50^{\circ}$ to minimize blockage, and tests were run with tilts of $0^{\circ}, 10^{\circ}, 20^{\circ}, 30^{\circ}, 40^{\circ}, 50^{\circ}$ (positive angles indicating tilt toward the airflow). Each combination of wind speed and tilt angle was tested in one test run lasting approximately 150 seconds.

After each test run, the percentage light transmission loss (TL\%) on soiled tape and soiled glass areas of the coupon were measured, and the difference in TL\% from clean tape and clean glass gave deposition and accumulation values, respectively. Because clean tape was not perfectly clear, deposition and accumulation measurements are not directly comparable. Dust mass was not measured, but to provide a sense of scale TL of $20 \%$ was roughly equivalent to surface load of 5 g.m ${ }^{-2}$. Transmission measurements were made at six locations along the coupon centerline: $2.5,5.5,8.5,11.5,14.5,17.5 \mathrm{~cm}$ from the coupon's windward edge. These spot measurements provided insight on how airflow field over 
131 the coupon affected particle deposition and detachment. The six spot measurements were then averaged to characterize overall deposition or accumulation on the coupon.

\subsection{Field Tests}

135 The wind-tunnel tests were replicated and expanded on by field tests at the QEERI Outdoor Test Facility, a semi-urban field station in Doha, Qatar. To isolate the effect of tilt angle on soiling it was necessary to minimize variation of other parameters in the field tests, especially wind direction and speed. To achieve this, a rotating wind vane was built (Figure 2), such that the coupons' tilt axis perpendicular to the wind. Coupons were exposed for periods of 4-8 hours during the daytime while wind speed was constantly recorded, and the data for the day was used if the median wind speed during the exposure was within 4-6 m.s ${ }^{-1}$. A minimum of three such days was obtained for each pair of tilt 142 angles.

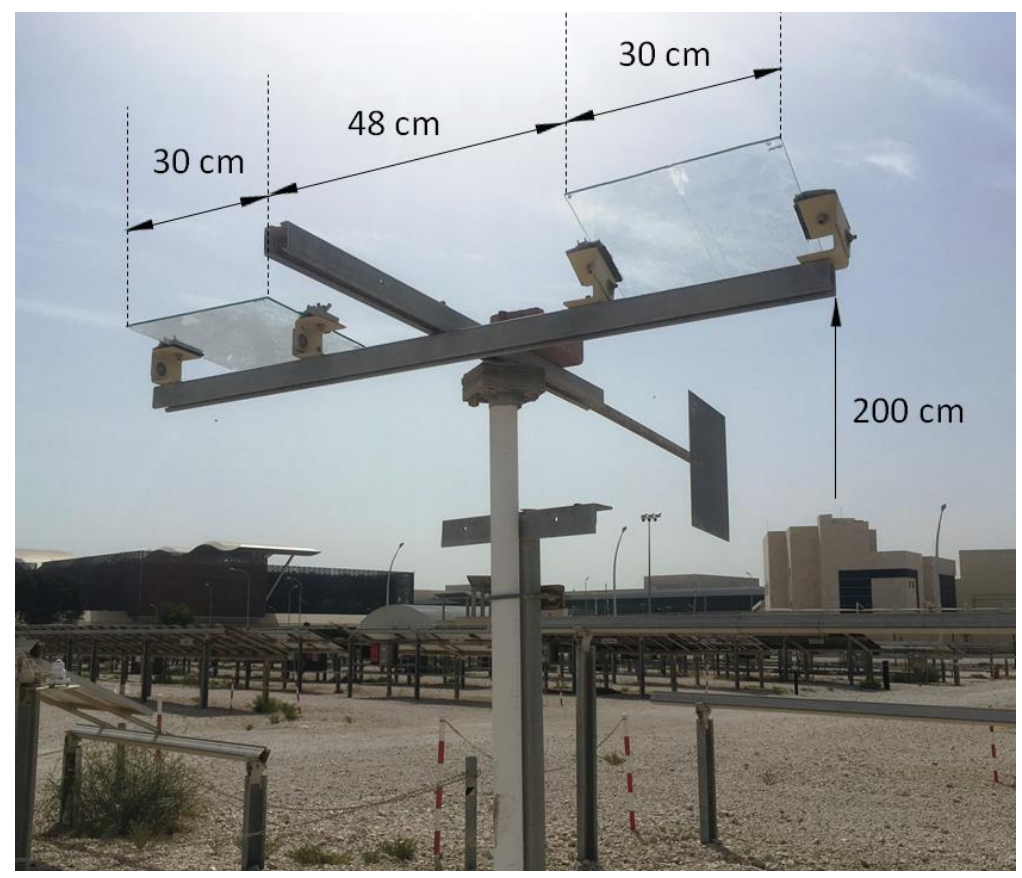

Figure 2. Field soiling wind vane in Doha. In shown example, left coupon is at $0^{\circ}$ tilt and the right at $-22^{\circ}$ (i.e. facing downwind, or

"backward"). Double-sided adhesive tape was attached along their centerlines (slightly visible on the right coupon).

In principle enough test days could be run to eliminate variation in other environmental parameters such as relative humidity (RH) and aerosol concentration (PM), however it may take years to obtain three days with similar values of all parameters. To minimize the effect of non-controlled parameters, coupons were tested in pairs at dissimilar tilts (method described further below). That is, within each 
test day the only variable was coupon tilt. Later analysis showed that the effect of tilt angle on deposition and accumulation was reasonably consistent between the three test days. This does not mean that RH and PM do not influence soiling; rather that their degree of influence was fairly similar at different tilt angles.

The wind vane held a pair of glass coupons, each $30 \times 30 \mathrm{~cm}$, at dissimilar tilt angles. Tilts were varied from $90^{\circ}$ (vertical, face toward the wind) to $-90^{\circ}$ (vertical, face downwind) in increments of $22.5^{\circ}$. Pairwise testing created a choice of protocols, e.g. (a) keep one coupon at a constant reference angle while varying the other $\left(0^{\circ}\right.$ vs $22^{\circ}, 0^{\circ}$ vs. $\left.45^{\circ}, \ldots\right)$, or (b) test sequential pairs of tilts $\left(0^{\circ}\right.$ vs $22^{\circ}, 22^{\circ}$ vs $45^{\circ}$, ...). We chose the latter method because it provided direct evidence of tilt angle that caused the extreme (least or most) deposition and accumulation. That is, each tilt angle being tested directly against its "neighbors" provided certainty as to where the peak occurred. However this approach sacrifices accuracy when comparing results at non-adjacent tilts, e.g. deposition at $45^{\circ} \mathrm{vs}-45^{\circ}$. This is because several intermediate results would need to be combined, compounding their uncertainties. As noted each pair of tilts was tested on at least three days, to evaluate consistency of the data and average it.

As in the wind tunnel tests, each coupon had a strip of double-sided adhesive tape along its stream-wise centerline, with the taped area used for deposition measurement and bare glass for accumulation measurement. In the field tests, coupons were imaged in the lab with an optical microscope and soiling was measured by the percentage of surface covered by particles. This was done at spots 5, 10, 15, 20 and $25 \mathrm{~cm}$ from the coupon's windward edge, along its centerline. The average of the five spot measurements on tape was taken, providing the deposition measurement. To aid interpretation, the deposition values were then normalized by the deposition at $0^{\circ}$ tilt. Normalized results are denoted with an apostrophe, e.g. deposition', a dimensionless fraction. The same procedure was used to calculate average accumulation and accumulation' on the coupon bare glass.

\subsection{CFD Model}

Computational fluid dynamic (CFD) simulation of the field tests was performed. The purpose of the CFD study was to see if trends in the experimental results could be explained by changes in airflow characteristics with coupon tilt. Dust deposition (impacting particles) was modeled but not accumulation, as it is difficult to accurately model particle adhesion and removal forces in natural conditions.

CFD simulations were performed with COMSOL Multiphysics ${ }^{\circledR}$ software, using its Turbulent Flow and Particle Tracing for Fluid Flow modules. Flow velocity was solved using a k- $\varepsilon$ turbulence model with wall function. The k- $\varepsilon$ model was chosen because it readily converges to a solution and its moderate accuracy is adequate for this study, while the wall function provides an adequate analytic solution for near-wall flows without large computational requirement. Default turbulence parameters and air physical properties were used. The model was run with the same coupon tilt angles as the field tests, at the single wind speed $5 \mathrm{~m} . \mathrm{s}^{-1}$. Particles were released at the inlet with the same velocity as the flow, at 
concentration $2.5 \times 10^{4}$ particles. $\mathrm{m}^{-1}$. Particles were assigned uniform density $2700 \mathrm{~kg} \cdot \mathrm{m}^{-3}$ and diameter $30 \mu \mathrm{m}$ (Figgis, 2018). Turbulent dispersion of particles was simulated using the built-in discrete random walk function. Particles impacting the top face of the coupons were "frozen" and counted to measure deposition. As mentioned, dust detachment was not modeled so there is no equivalent CFD measure of accumulation. Further details of the CFD model geometry, inlet velocity profile, and evaluation of model quality are provided in the Supplementary Information.

Average deposition was characterized by the number of particle impacts on the upper face of the coupons, as determined by the software's particle tracing feature. Impacts within $5 \mathrm{~cm}$ of the windward and leeward edges were excluded to simulate the experimental methods, and to avoid bias from the large amount of deposition at the windward edge which was observed in the experimental and CFD studies.

\section{Results and Discussion}

\subsection{Wind Tunnel}

Wind-tunnel results of coupon deposition (average of the five spot measurements) versus tilt angle and wind speed are shown in Figure 3a. There is significant interaction between the parameters: At low wind speed (1 m.s-1) deposition roughly decreased with tilt angle, whereas at high wind speed (3-4 m.s $\left.{ }^{1}\right)$ it is increased with tilt. In between $\left(2 \mathrm{~m} \cdot \mathrm{s}^{-1}\right)$, deposition peaked in a band of $10-40^{\circ}$ tilt.

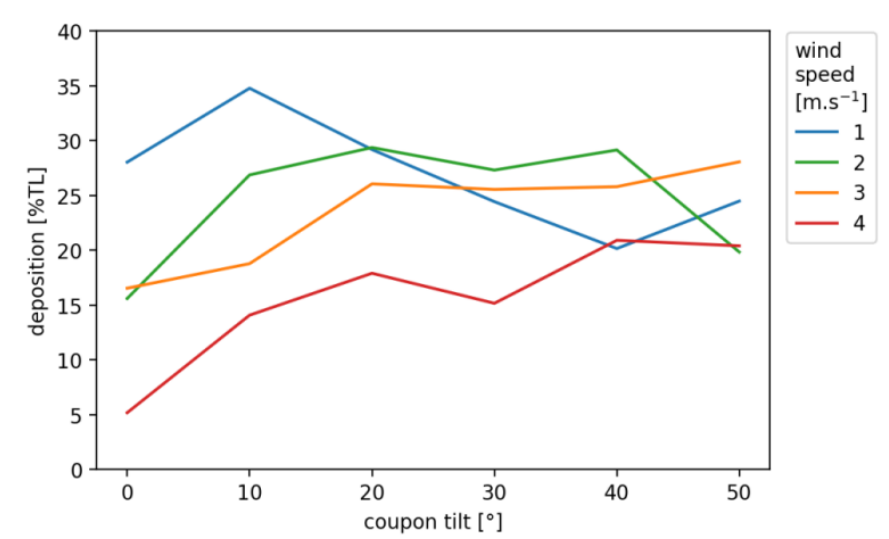

(a)

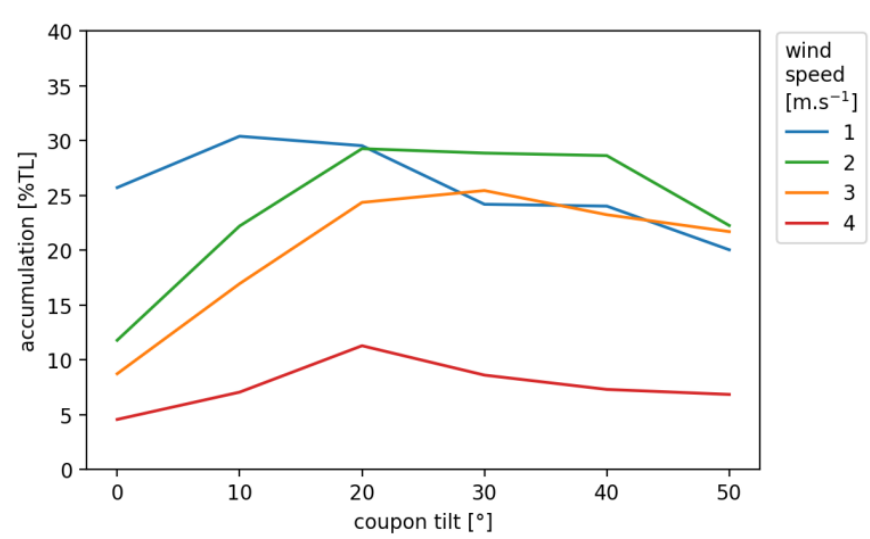

(b)

Figure 3. Wind-tunnel results of (a) deposition and (b) accumulation on coupon vs. wind speed and tilt angle

The reversal of the deposition vs. tilt response, depending on wind speed, can be explained by aerosol mechanics. At $1 \mathrm{~m} \cdot \mathrm{s}^{-1}$ wind speed, deposition was dominated by gravity so it roughly followed the cosine of coupon tilt. In contrast at 3-4 m.s $\mathrm{s}^{-1}$ inertial deposition dominated, that is the dust particles (mostly tens of microns in diameter) had sufficient inertia to deviate from sharply-turning flow streamlines near the coupon surface and impact it (Aluko and Noll, 2006). As coupon tilt increased the 
213 incidence angle of particles to the surface increased, causing them to deviate from flow streamlines and 214 deposit.

215 Unexpectedly, the amount of deposition on the surface generally decreased with higher wind speed, 216 especially at lower tilt angles. With constant dust concentration and size distribution, the mass 217 deposition rate of aerosol particles conventionally increases with wind speed (Figgis et al., 218 2017a)(Goossens et al., 1993). Closer inspection of the coupons provided an explanation of the present 219 results by the soiling layer structure, i.e. the geometric configuration of particles and voids in the layer (Goossens and van Kerschaever, 1999). Faster wind created more voids in the soiling layer, while slower wind resulted in a denser structure. The voids transmit light and thus decrease the apparent deposition (as measured by TL\% of the adhesive tape). This phenomenon was most pronounced at low tilt angles, although at almost all angles the least deposition (TL\%) occurred at the highest wind speed. Another possibility considered was that as dust builds up on the tape it traps particles less efficiently, however this would attenuate but not reverse the expected deposition vs. wind speed response.

Wind-tunnel accumulation results are shown in Figure 3b. (As mentioned, accumulation TL\% could be higher than deposition TL\% for the same test because the adhesive tape was not perfectly clear.) Again, there is interaction between tilt angle and wind speed effects, although in a more complicated pattern than for deposition. At $1 \mathrm{~m} \cdot \mathrm{s}^{-1}$ accumulation generally decreased with tilt angle. At 2-3 m.s ${ }^{-1}$ it exhibited a broad peak across the tilt range $20-40^{\circ}$. At $4 \mathrm{~m} \cdot \mathrm{s}^{-1}$ the quantity of accumulation decreased dramatically, and it exhibited a maximum at $20^{\circ}$ tilt.

The accumulation results can be understood from particle deposition mechanics, and also previous studies of particle detachment. At wind speed $1 \mathrm{~m} \cdot \mathrm{s}^{-1}$, there is little particle detachment and accumulation (like deposition) is dominated by gravity. At $3 \mathrm{~m} \cdot \mathrm{s}^{-1}$ there is a small decrease in accumulation at all tilts, and a dramatic decrease at $4 \mathrm{~m} . \mathrm{s}^{-1}$. This is consistent with previous studies showing increasing particle rebound with flow velocity (Figgis et al., 2018), and the existence of a threshold flow velocity above which resuspension occurs (Figgis et al., 2017a)(Ilse et al., 2018). The present results suggest that together these mechanisms start at wind speed around $3 \mathrm{~m} \cdot \mathrm{s}^{-1}$ and become pronounced around $4 \mathrm{~m} \cdot \mathrm{s}^{-1}$.

Consider now variation of deposition and accumulation along coupons' stream-wise centerline, which may provide insight on flow and particle dynamics. Deposition distributions are shown in Figure 4a, for the case of $4 \mathrm{~m} \cdot \mathrm{s}^{-1}$ wind speed. (This wind speed is most comparable with field tests; distributions at other wind speeds are presented in the Supplementary Information.) At $0-10^{\circ}$ tilt deposition generally increased with distance from the windward edge, whereas at steeper tilts (up to $50^{\circ}$ ) it gradually decreased. That is, steepening the tilt angle generally moved the point of maximum deposition toward the coupon's leading edge. Variation of dust accumulation along the coupon (Figure $4 b$ ) is similar to that of deposition, i.e. steepening the tilt angle brought forward the point of maximum accumulation. 


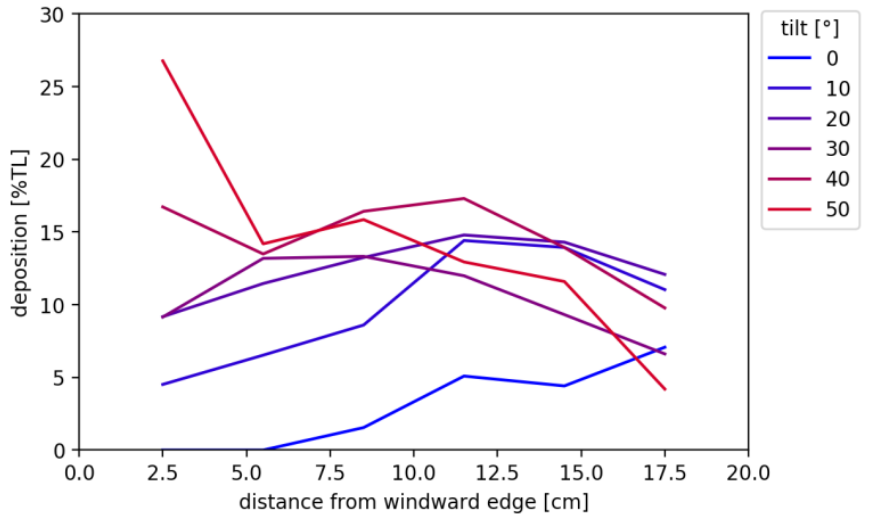

(a)

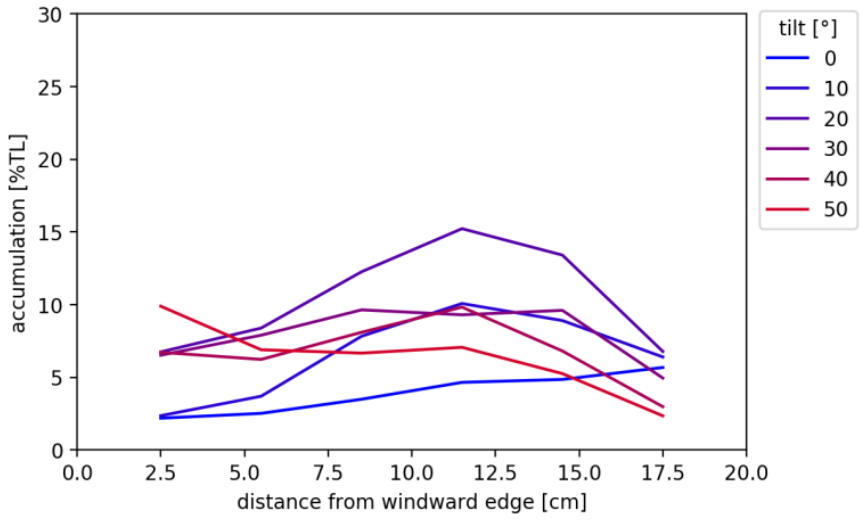

(b)

Figure 4. Wind-tunnel results of (a) deposition and (b) accumulation on coupon vs. coupon position (stream-wise centerline) and tilt angle, wind speed $4 \mathrm{~m} \cdot \mathrm{s}^{-1}$

Figure 5 shows the structure of the deposition and accumulation soiling layers on a sample coupon in detail. The upper two strips are original photos of the layers, in the lower strips the contrast was increased to better show the structure. There is greater granularity in the accumulation layer than the deposition layer, indicating that resuspension is a spatially non-uniform process: even at a small scale, some locations are subjected to more (or less) dust resuspension than their neighbors.

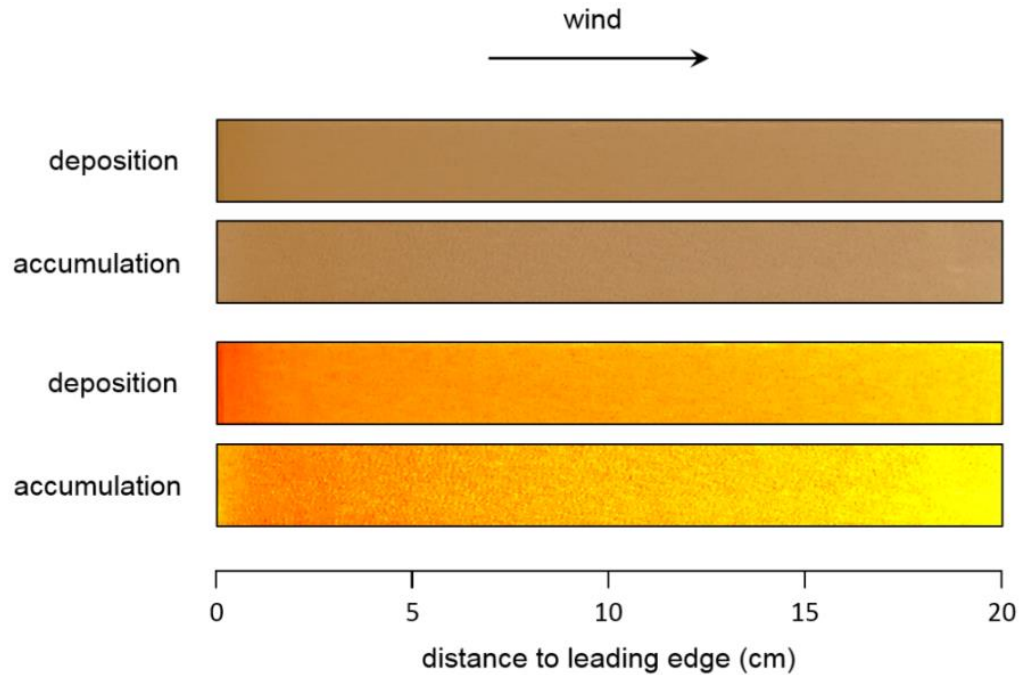

Figure 5. Structure of soiling layer along a coupon in wind tunnel (wind speed $2 \mathrm{~m} . \mathrm{s}^{-1}$, coupon tilt $30^{\circ}$ ). Each strip in the figure is $2 \mathrm{~cm}$ wide (vertical direction as shown). Top pair: raw photographs. Bottom pair: Increased contrast, revealing granularity of accumulation.

260 To summarize the wind-tunnel results: Deposition tended to increase with coupon tilt angle (from $0^{\circ}$ to $26150^{\circ}$ ) due to inertial particle impacts, except in very light wind when gravity dominated. Deposition 
apparently decreased with wind speed, however this was due to looser structure of the soiling layer while using light transmission-based measurements. Accumulation tended to increase with coupon tilt up to $20-30^{\circ}$, and decrease at steeper tilts. It decreased greatly when wind speed reached $4 \mathrm{~m} . \mathrm{s}^{-1}$, as particles started to detach from the coupons again after impact.

\subsection{Field Results}

Deposition' and accumulation' results from the field tests are shown in Figure 6. (To recap, apostrophes denote the values were normalized by that at $0^{\circ}$ tilt). The plot is constructed by linking together deposition results for sequential pairs of tilts. For example, deposition at $22^{\circ}$ tilt was 1.38 times that at $0^{\circ}$, and deposition at $45^{\circ}$ was 1.04 times that at $22^{\circ}$. Hence deposition' at the $22^{\circ}$ abscissa is 1.38 , and at $45^{\circ}$ it is $1.38 \times 1.04=1.44$. As noted, pair-wise testing robustly identifies at which tilts peak deposition and accumulation occur, but this "linking" approach compounds uncertainty between non-adjacent tilts.

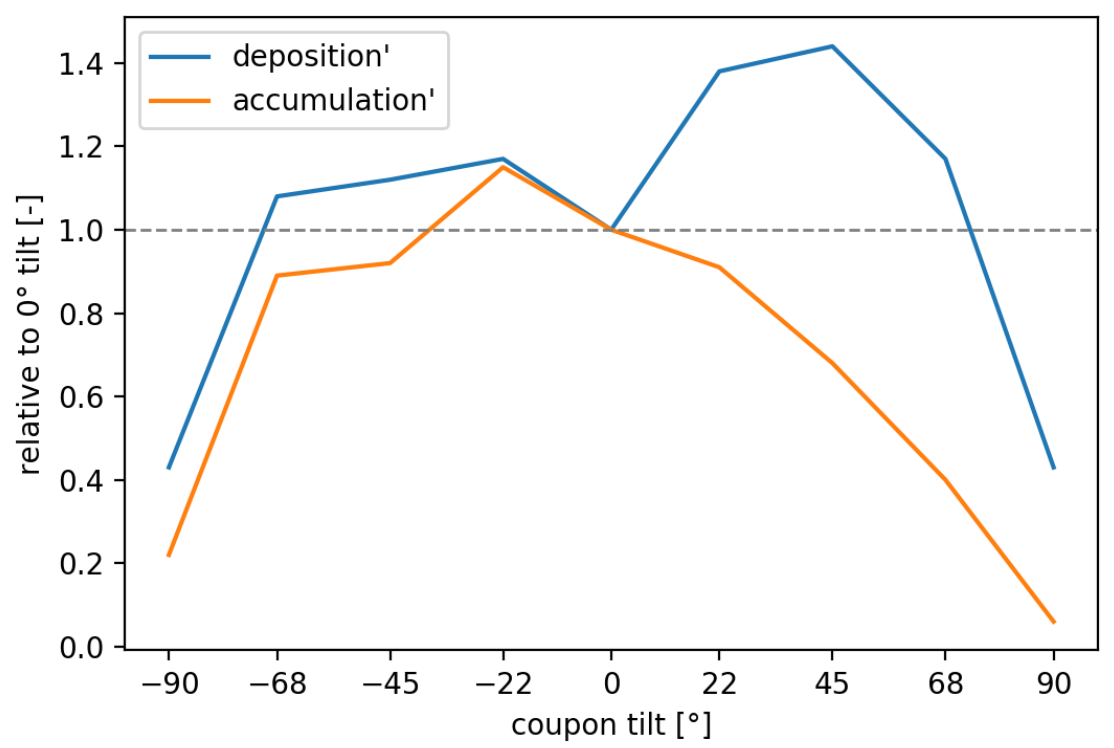

Figure 6. Field results of deposition' and accumulation' vs. coupon tilt. Values normalized by $0^{\circ}$ tilt. Plotted values are averages of three test days, each of which had median wind speed 4-6 m.s ${ }^{-1}$

Key observations from field results of Figure 6 are:

- Maximum deposition occurred when coupons were tilted at $45^{\circ}$ (toward the wind)

- Maximum dust accumulation occurred at $-22^{\circ}$ tilt (away from the wind)

- Tilting toward the wind generally resulted in more deposition than the same angle away from the wind 
- There was a deposition "valley" at $0^{\circ}$, i.e. tilting slightly either toward or away from the wind created more deposition than horizontal

- Little dust accumulates on vertical coupons, facing either way.

To further study the concept of stowing PV modules vertically at night, we conducted a brief additional experiment and applied double-sided tape to both sides of a vertical coupon for one test day. It was found that the downwind face had 2.50 times the dust accumulation of the windward face, but only 0.39 times as much deposition. That is, more particles impacted the windward side but they tended to be blown off again, whereas the (fewer) particles impacting the downwind side were protected from detachment. Wind characteristics on the two sides are discussed in the CFD section.

Such dust detachment (from bare glass) helps explain the interesting divergence of deposition and accumulation at forward tilts in Figure 6. Tilting from horizontal to $45^{\circ}$ increased deposition but decreased accumulation - i.e. in this region soiling behavior was governed by particle detachment. Tilting further, deposition decreased faster than accumulation-i.e. here soiling behavior was governed by the number of particle impacts, while detachment became less relevant.

It is tempting to interpret the large difference between deposition and accumulation at $45^{\circ}$ tilt as evidence that this angle maximizes cleaning by perpendicular wind. However it might have been due to dust particles rebounding (never adhering to the surface) rather than resuspending (removal of resident particles from the surface, i.e. cleaning). To robustly determine the optimum tilt for wind cleaning, one would conduct experiments with already-soiled coupons, rather than clean coupons as in the present study. Zimon (Zimon, 1969) reported such an experiment using 20-30 $\mu \mathrm{m}$ glass spheres resting on a steel surface; the resulting "adhesion rosette" showed greatest resuspension at $30^{\circ}$ forward tilt. The subject is discussed further in the CFD analysis.

Field studies of PV soiling universally report greatest soiling at zero tilt (Figgis et al., 2017a). At face value, the present finding of maximum accumulation at $-22^{\circ}$ (downwind) tilt appears to contradict that. However, the difference can be explained by field studies typically having exposure periods of multiple days and fixed orientations, resulting in (i) substantial periods without wind (whereas this study focused on wind speed around $5 \mathrm{~m} \cdot \mathrm{s}^{-1}$ ), and (ii) wind direction changing with respect to the fixed tilted collector (whereas this study used a wind vane mounting). Thus it is compatible to observe greatest soiling at $22^{\circ}$ tilt during periods of perpendicular wind, yet greatest soiling at horizontal tilt over several days.

In similar conditions of wind speed and tilt angle, the field results were similar to the wind tunnel for deposition (compare Figure 6 to Figure 3a), but were somewhat inconsistent for accumulation (Figure $3 \mathrm{~b}$ ). The wind tunnel generally produced an accumulation peak around $20^{\circ}$ tilt, depending on wind speed, whereas the field results showed monotonic decrease of accumulation with forward tilt. We speculate that occasional gusts of strong wind occurred during the field tests, which removed "extra" dust than the median wind speed. Of course such gusts did not occur during the controlled wind-tunnel 
tests. Alternatively, differences in conditions and methods between the wind tunnel and field tests (e.g. relative humidity, soiling measurement technique) may have contributed to the inconsistent result.

Like the wind-tunnel tests, variation of deposition and accumulation along coupons was analyzed in the field tests. For each coupon tilt, the test day with median wind speed closest to $4 \mathrm{~m} . \mathrm{s}^{-1}$ was used for this analysis. Because ambient dust concentration varied between test days, to enable comparison each spot measurement was normalized by the average deposition on the coupon for that day. Vertical tilts $\left(-90^{\circ}\right.$ and $90^{\circ}$ ) are omitted because their dust distributions were highly irregular, making the plots difficult to read. The results are shown Figure 7a. Generally, when coupons were tilted toward the wind (positive tilts) dust deposition was concentrated at the windward edge and decreased along the coupon. The reverse occurred when coupons were tilted away from the wind or horizontally - deposition increased along the coupon. Where their test conditions overlapped, the field and wind-tunnel results (Figure 4a) were broadly consistent, both showing increasing deposition along the coupon at $0^{\circ}$ tilt, and decreasing

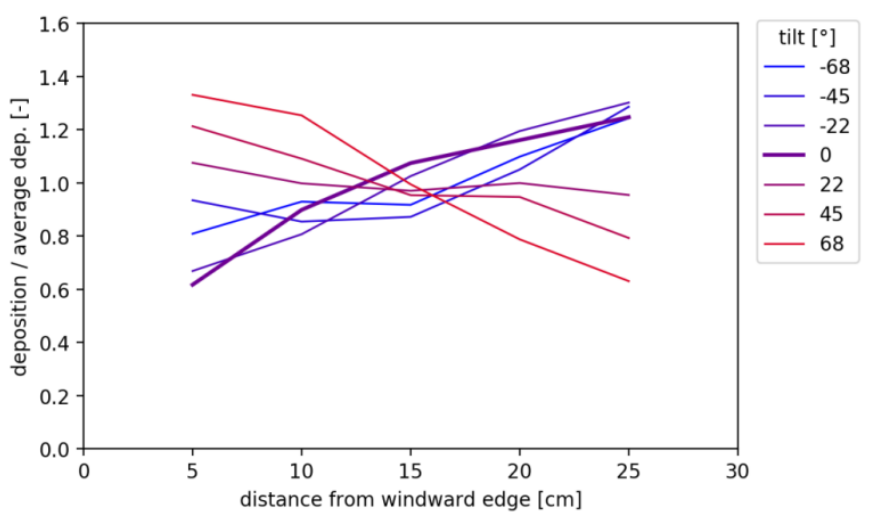

(a)

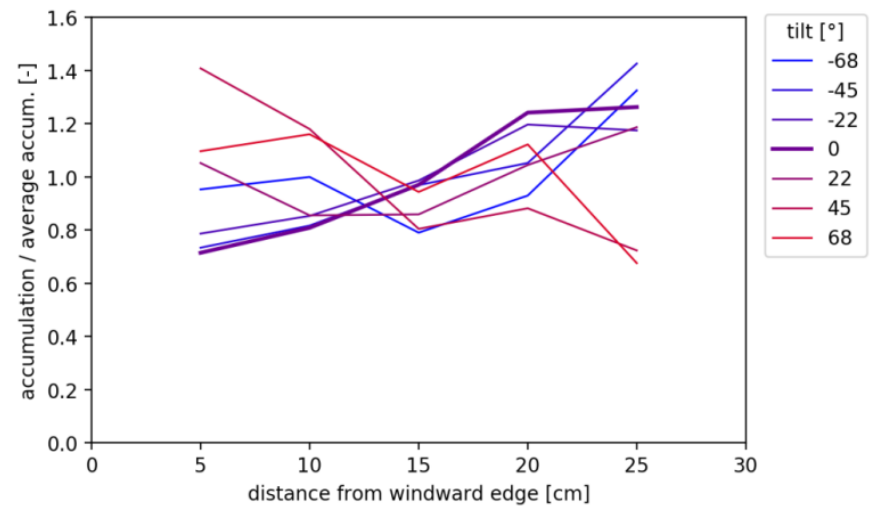

(b)

Figure 7. Field results of (a) deposition and (b) accumulation vs. location on coupon and tilt angle, wind speed $\sim 4$ m.s $\mathrm{s}^{-1}$ Values normalized by average deposition on the coupon

Variation of accumulation along the field coupons is shown in Figure 7b. Although there is much scatter in the data, the overall trends are roughly similar to deposition (Figure 7a): increasing accumulation along the coupon from backward tilts up to $22^{\circ}$ forward, and decreasing accumulation at steeper forward tilts. The field results are roughly consistent with those from the wind-tunnel (Figure $4 b$ ), except that in the field, peak accumulation usually occurred at the edges of coupons rather than their middles. 


\subsection{CFD Results}

343 CFD prediction of deposition (normalized by the value at $0^{\circ}$ tilt) versus coupon tilt is shown in Figure 8 . 344 As discussed earlier, the main deposition mechanisms for particles tens-of-microns in diameter are gravity and inertial impacts (particles separating from flow that is abruptly deflected by the coupon). Therefore one may expect deposition to be predicted by flow velocity component normal to the surface, also plotted in Figure 8. This parameter, $\mathrm{u}_{\text {normal' }}$, was obtained from CFD results of flow velocity normal to the surface at a distance of $10^{-4} \mathrm{~m}$ (similar results were obtained at other $\leq 1 \mathrm{~mm}$ distances), averaged over the length of coupon between the $5 \mathrm{~cm}$ and $25 \mathrm{~cm}$ positions, normalized by its value at $0^{\circ}$ tilt. The plot shows that in the CFD model, deposition was fairly well predicted by $\mathrm{u}_{\text {normal }}$ except for divergence at steep forward tilts.

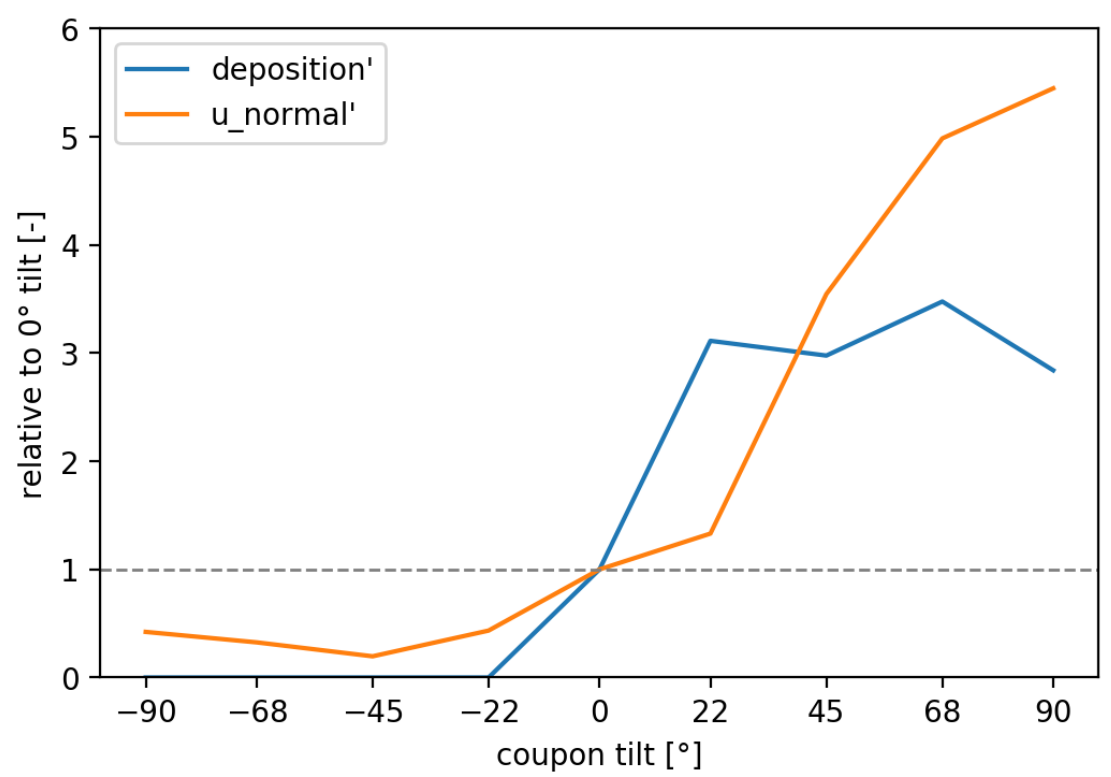

Figure 8. CFD results of deposition' and $\mathrm{u}_{\text {normal }}$ vs. coupon tilt angle, wind speed $5 \mathrm{~m} \cdot \mathrm{s}^{-1}$. Values normalized by $0^{\circ}$ tilt. Deposition' is zero from $-90^{\circ}$ to $-22^{\circ}$ tilt

At forward tilts, the CFD deposition results (Figure 8) are roughly consistent with the field results (Figure 6), although the scale of variation is greater in CFD. At backward tilt however, the CFD model predicted zero deposition whereas it remained substantial in the field tests. Subsequent to this study further tests were performed in the same wind tunnel (Goossens, 2019), using coupon tilts from $-90^{\circ}$ to $90^{\circ}$ in wind speed of $3 \mathrm{~m} . \mathrm{s}^{-1}$. Those results (Figure 9) were consistent with field and CFD results at forward tilts, and between the field and CFD results at backward tilts. The range of results at backward tilts is attributable to flow turbulence, which would have been relatively subdued in the CFD model and wind tunnel and relatively great in the field. 


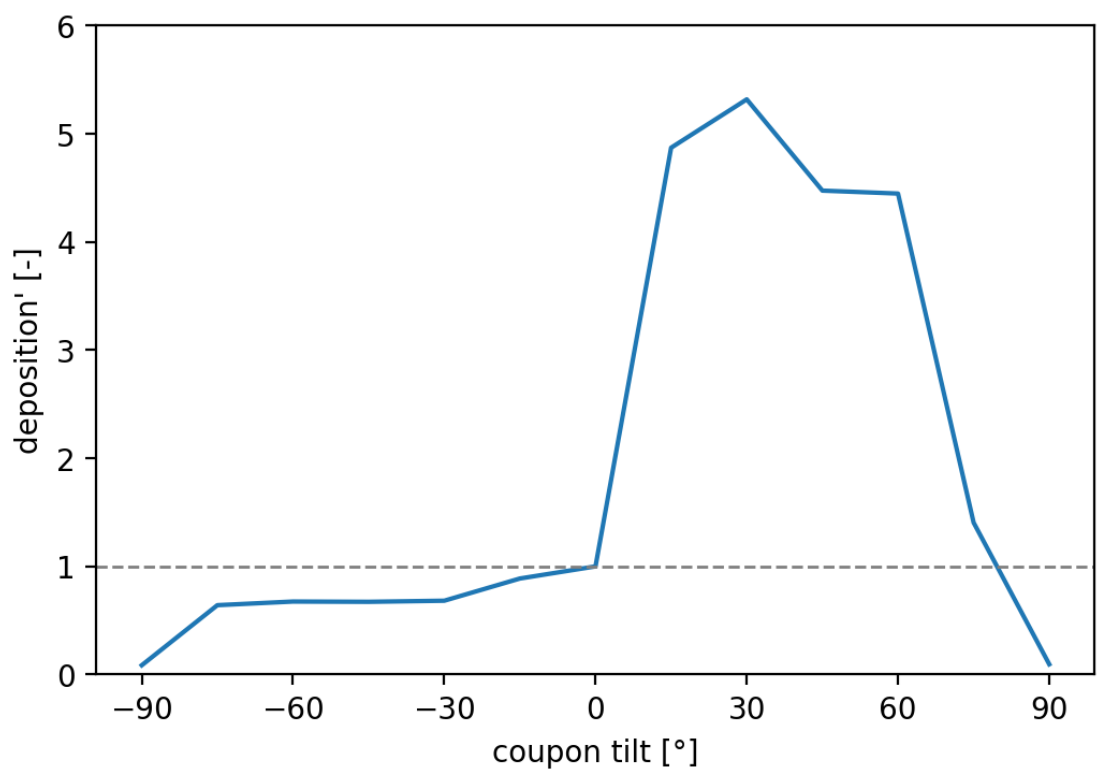

Figure 9. Wind-tunnel results of deposition' vs. coupon tilt angle, wind speed $3 \mathrm{~m} \cdot \mathrm{s}^{-1}$. Values normalized by $0^{\circ}$ tilt. (Goossens, 2019)

The CFD model was not used to simulate particle detachment, as mentioned, but was used to calculate airflow properties that might explain field results of dust accumulation. Two flow parameters were considered: velocity tangential to the coupon ( $\mathrm{u}_{\text {tangent }}$ ) at $10^{-5} \mathrm{~m}$ from the wall (the length scale of relevant dust particles), and flow shear rate (rate of change of $\mathrm{u}_{\text {tangent }}$ with distance from the wall) at the wall itself. These parameters were averaged between the $5 \mathrm{~cm}$ and $25 \mathrm{~cm}$ positions on the coupon.

374 Variations of $\mathrm{u}_{\text {tangent }}$ and wall shear rate, normalized by their values at $0^{\circ}$ tilt, are shown in Figure 10.

375 The two parameters show similar trends, with distinct maxima around $22^{\circ}$ tilt. This peak can be 376 understood in terms of competition between obstruction to the wind and spacing of flow streamlines near the wall: As forward tilt angle increases, the coupon obstructs and slows the approaching wind (an adverse pressure gradient is created), but the tilt also causes flow energy to be transferred to (and thus accelerate) fluid layers close to the wall. The CFD model indicates that at around $22^{\circ}$ tilt, the balance between these effects results in relatively high flow speed and shear rate near the surface. 


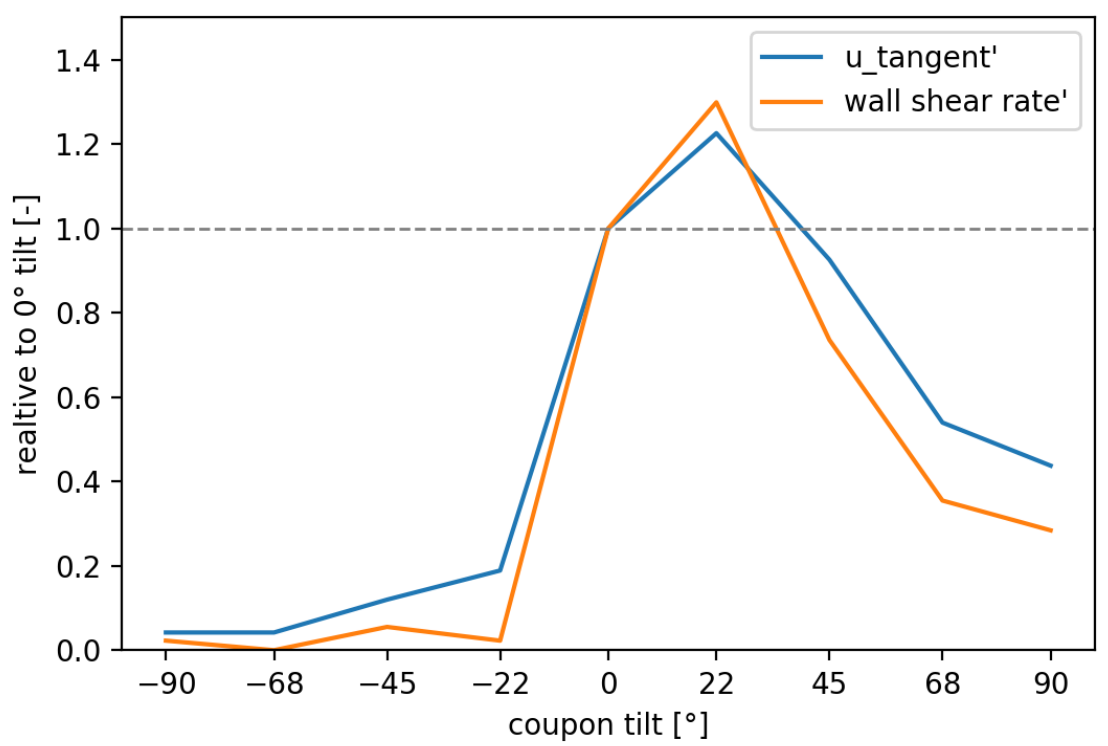

Figure 10. CFD results of $\mathrm{u}_{\text {tangent }}(10 \mu \mathrm{m}$ from surface $)$ and wall shear rate vs. coupon tilt angle, wind speed $5 \mathrm{~m} . \mathrm{s}^{-1}$. Values normalized by $0^{\circ}$ tilt

The CFD results may raise the expectation that tilt around $22^{\circ}$ would cause greatest dust detachment from a soiled surface in perpendicular wind. However the experiments herein found the largest difference between deposition and accumulation occurred at $45^{\circ}$ tilt (field tests) or $50^{\circ}$ (wind tunnel tests). Recall also the pertinent report (Zimon, 1969) that maximum detachment of glass spheres from steel plates occurred at $30^{\circ}$ tilt (the experiment used tilt increments of $30^{\circ}$, so in fact the maximum could have occurred anywhere from $0^{\circ}$ to $60^{\circ}$ ). Overall this collection of research indicates that dust resuspension is maximized by tilting a surface in the region of $30-50^{\circ}$ toward the wind.

Soiling is a balance between dust deposition and detachment, and the present results (experimental and CFD) show how the balance changes with coupon angle: Tilting moderately toward the wind $\left(22-45^{\circ}\right)$ increases dust deposition but - to an even greater extent - increases its detachment by fast near-wall flow. Tilting more steeply $\left(68-90^{\circ}\right)$ retards airflow approaching the coupon which reduces dust detachment but - to an even greater extent - suppresses its deposition. So overall, dust accumulation in the field decreases as the surface is progressively tilted toward the wind (Figure 6).

\section{Conclusions}

401 Dust deposition (all particles impacting the surface) and accumulation (particles remaining on the surface) were measured on glass coupons, at various tilt angles perpendicular to the wind. Experiments were performed in a wind tunnel with dust generator, and in the field in Qatar using a rotating wind vane. Airflow characteristics over the coupons were simulated using CFD. 
Regarding dust depositions, all methods (experimental and CFD) demonstrated that deposition increased as the coupon was progressively tilted from $0^{\circ}$ (horizontal) to roughly $50^{\circ}$ (toward the wind). At steeper forward tilts in the field tests, deposition then decreased sharply again, which was not predicted by the CFD model. Tilting the coupon backward (away from the wind) produced a range of results across the study methods, with zero deposition predicted by CFD yet substantial amounts observed in the field. The differences were attributable to differences in turbulence levels between the methods.

Effect of wind speed on deposition was investigated by controlled wind-tunnel tests. Increasing wind speed at the same coupon tilt angle decreased the measured deposition, against expectation. Further analysis suggested this was due to creation of voids in the soiling layer at higher wind speeds, lowering the deposition value which was quantified by loss in transmission.

Regarding dust accumulation, in field tests it reached a maximum at $-22^{\circ}$ tilt (away from the wind), and from there decreased monotonically with tilt angle in either direction. Generally, there was less accumulation at forward tilt than backward tilt of the same angle magnitude. In particular, at $90^{\circ}$ (vertical, facing the wind) there was only $40 \%$ as much accumulation as $-90^{\circ}$ (vertical, facing downward). Somewhat different results were observed in the wind tunnel: Going from $0^{\circ}$ to $30^{\circ}$ tilt caused accumulation to increase, especially at low wind speed $\left(\leq 3 \mathrm{~m} \cdot \mathrm{s}^{-1}\right)$. It is thought the difference was due to greater turbulence and gusts of natural wind compared to wind-tunnel flow. This ambiguous realm encourages further field tests in a wider variety of wind speeds.

The motivation for this study was to better understand how wind affects soiling of PV systems, in particular whether 1-axis trackers could be used to combat soiling in windy conditions. In this regard the key findings were: dust accumulation on wind-facing coupons decreased with faster wind speed and steeper tilt; maximum accumulation occurred when the coupon was tilted slightly away from the wind ($22^{\circ}$ ); and tilting toward the wind resulted in less soiling than the same angle away from the wind. This suggests that for existing commercial trackers, which typically rotate through $\pm 45^{\circ}$ or $\pm 60^{\circ}$, soiling reduction could be achieved "for free" on windy nights by stowing them at maximum tilt toward the wind. Very little soiling could be achieved if they were stowed vertically toward the wind.

\section{Acknowledgments}

This publication was made possible in part by an award (NPRP 7-987-2-372) from the Qatar National Research Fund, a member of Qatar Foundation. The statements made herein are solely the responsibility of the authors.

\section{References}

Aluko, O., Noll, K.E., 2006. Deposition and Suspension of Large, Airborne Particles. Aerosol Sci. Technol. 40, 503-513. https://doi.org/10.1080/02786820600664152 
Biryukov, S., 1998. An experimental study of the dry deposition mechanism for airborne dust. J. Aerosol Sci. 29, 129-139.

Costa, S.C.S., Sonia, A., Diniz, A.C., Kazmerski, L.L., 2016. Dust and soiling issues and impacts relating to solar energy systems : Literature review update for $2012-2015$. Renew. Sustain. Energy Rev. 63, 33-61. https://doi.org/10.1016/j.rser.2016.04.059

Figgis, B., 2018. Investigation of PV Soiling and Condensation in Desert Environments via Outdoor Microscopy. University of Strasbourg.

Figgis, B., Ennaoui, A., Ahzi, S., Rémond, Y., 2017a. Review of PV soiling particle mechanics in desert environments. Renew. Sustain. Energy Rev. 76, 872-881. https://doi.org/10.1016/j.rser.2017.03.100

Figgis, B., Guo, B., Javed, W., Ahzi, S., Rémond, Y., 2018. Dominant environmental parameters for dust deposition and resuspension in desert climates. Aerosol Sci. Technol. 52, 788-798. https://doi.org/10.1080/02786826.2018.1462473

Figgis, B., Guo, B., Javed, W., Ilse, K., Ahzi, S., Rémond, Y., 2017b. Time-of-day and exposure influences on PV soiling, in: IRSEC 2017. Tangier.

Fryrear, D.W., 1986. A field dust sampler. J. Soil Water Conserv. 41, 117-120.

Goossens, D., 2019. Soiling of Photovoltaic Modules: Size Characterization of the Accumulated Dust. J. Clean Energy Technol. 7, 25-31. https://doi.org/10.18178/jocet.2019.7.3.505

Goossens, D., Offer, Z.Y., Zangvil, A., 1993. Wind tunnel experiments and field investigations of eolian dust deposition on photovoltaic solar collectors. Sol. Energy 50, 75-84. https://doi.org/10.1016/0038-092X(93)90009-D

Goossens, D., van Kerschaever, E., 1999. Aeolian Dust Deposition on Photovoltaic Solar Cells: the Effects of Wind Velocity and Airborne Dust Concentration on Cell Performance. Sol. Energy 66, 277-289.

Ilse, K.K., Figgis, B.W., Naumann, V., Hagendorf, C., Bagdahn, J., 2018. Fundamentals of soiling processes on photovoltaic modules. Renew. Sustain. Energy Rev. 98, 239-254. https://doi.org/10.1016/j.rser.2018.09.015

Javed, W., Guo, B., Figgis, B., 2017. Modeling of photovoltaic soiling loss as a function of environmental variables. Sol. Energy 157, 397-407. https://doi.org/10.1016/j.solener.2017.08.046

Lu, H., Lu, L., Wang, Y., 2016. Numerical investigation of dust pollution on a solar photovoltaic (PV) system mounted on an isolated building. Appl. Energy 180, 27-36. https://doi.org/10.1016/j.apenergy.2016.07.030

Lu, H., Zhang, L.Z., 2019. Influences of dust deposition on ground-mounted solar photovoltaic arrays: A CFD simulation study. Renew. Energy 135, 21-31. https://doi.org/10.1016/j.renene.2018.11.096

Lu, H., Zhao, W., 2018. Effects of particle sizes and tilt angles on dust deposition characteristics of a ground-mounted solar photovoltaic system. Appl. Energy 220, 514-526. 
476 https://doi.org/10.1016/j.apenergy.2018.03.095

477 Péwé, T.L., 1981. Desert Dust: Origin, Characteristics, and Effect on Man. Geol. Soc. Am.

478 Smits K., Goossens, D., 1995. Wind tunnel simulations of aeolian dust deposition on thermic solar 479 collectors. Appl. Sol. Energy 30, 75-89.

480 Zimon, A.D., 1969. Adhesion of Dust and Powder. Spring Science and Business Media. 